\title{
Le jour où le Poète reprit sa lyre. " Down by the Salley Gardens » ou la nostalgie des origines
}

\author{
Anne-Marie GOUIFFÈS (Paris) ${ }^{1}$
}

\section{Summary}

The Irish poet Yeats tells the story that as he was walking one day in the Ballisodare countryside, he heard a woman singing some remnants of a very old song that moved him. The poem that he then wrote is, he assures us: "an attempt to reconstruct an old song from three lines imperfectly remembered by an old peasant woman in the village of Ballisodare, Sligo, who often sings them to herself" (Yeats 1889, 90).

Weaving together reverie and thought in "Down by the Salley Gardens", this poem of Yeats that became, in its turn a song again, I have tried to approach this land where poetry and music are still reaching out for each other, animated by an ever present longing for a common root: that of a time where words and sounds were not yet divided.

William Butler Yeats est de retour à Ballisodare ${ }^{2}$ comté de Sligo, berceau de sa famille maternelle. Il a vingt-deux ans, vingt-trois peut-être. Il a déjà publié quelques textes dans la Dublin University Review mais il n'a pas encore rencontré Maud Gonne, future égérie de la cause irlandaise, qui sera sa muse et son tourment. L'histoire pourrait commencer ainsi : tandis qu'il marche dans la campagne, il perçoit les bribes d'un air ancien. Il en est si troublé qu'il interrompt sa promenade pour rapporter ce qu'il vient d'entendre, et qu'il ne veut pas perdre. Yeats écrit en anglais. Un poème prend forme.

Celui-ci devra être compris, précise-t-il, comme " an attempt to reconstruct an old song from three lines imperfectly remembered by an old peasant woman in the village of Ballisodare, Sligo, who often sings them to herself $»^{3}$ (Yeats 1889, 90). Il y parvient si bien qu'en 1909, un compositeur décide d'adapter une mélodie aux vers de Yeats, ce qui les rend plus célèbres encore. D'autres suivront son exemple. L'histoire de "Down by the Salley Gardens ", poème redevenu chant, est dès lors une histoire inachevée qui invite à remonter à la source. 


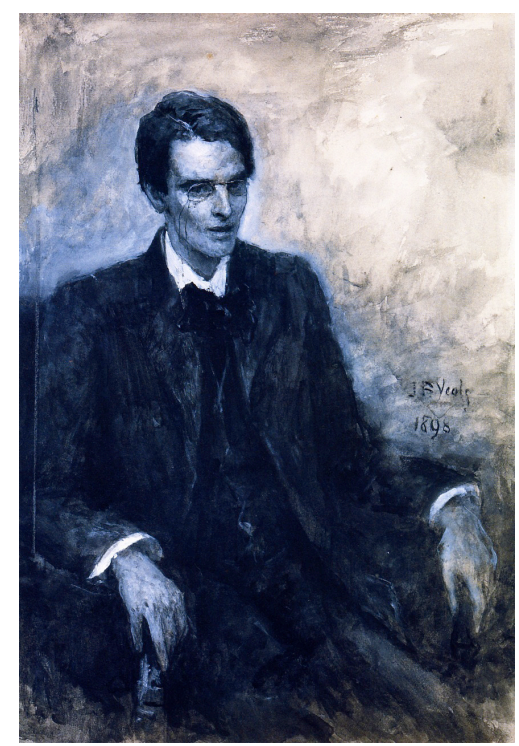

Figure 1. Portrait de William B. Yeats par son père John B. Yeats, 1898.

Graphite et lavis $(35,6 \times 25,3 \mathrm{~cm})$, National Gallery of Ireland

\section{Genèse d'un texte}

The Wanderings of Oisin and Other Poems ( Les errances d'Oisin et autres poèmes »), premier recueil de Yeats publié en 1889, contient un texte qui, au commencement, n'eut pas d'autre musique que celle de la langue anglaise :

Down by the salley gardens, my love and I did meet;

She passed the salley gardens, with little snow-white feet.

She bid me take love easy, as the leaves grow on the tree;

But I was young and foolish, with her would not agree.

In a field by the river, my love and I did stand,

And on my leaning shoulder, she laid her snow-white hand.

She bid me take life easy, as the grass grows on the weirs;

But I was young and foolish, and now am full of tears.

Lorsque Yeats publie Poems en 1895, il rassemble dans ce livre "The Wanderings of Ushee ", poème épique en trois parties, deux pièces : "The Countess Cathleen " et "The Land of Heart's Desire », et deux cycles de poèmes, "The Rose » et « Crossways ». Ce dernier cycle comporte une précision de date (1889) qui laisse à penser qu'il s'agit d'une réédition partielle de son tout premier recueil. 


\section{"An Old Song Re-Sung "}

Si les racines de "Crossways " ( La croisée des chemins ») remontent bien à l'opus de 1889, il ne s'agit pourtant pas d'une réédition pure et simple de "The Wanderings of Oisin ", mais d'une version à la fois remaniée et concentrée de sa première œuvre poétique. On le voit bien ici :

\section{The Wanderings of Oisin and Other Poems (1889)}

1. ["The Wanderings of Oisin "]

2. ["Time and the Witch Vivien " ${ }^{5}$

3. "The Stolen Child"

4. "Girl's Song "

5. "Ephemera"

6. "An Indian Song"

7. «Kanva, the Indian, on God"

8. "Kanva on Himself»

9. "Jealousy"

10. "Song of the Last Arcadian"

11. "King Goll»

12. "The Meditation of the Old Fisherman"

13. "The Ballad of Moll Magee "

14. "The Phantom Ship "

15. "A Lover's Quarrel among the Fairies"

16. "Mosada"

17. "How Ferencz Renyi Kept Silent"

18. "The Fairy Doctor"

19. "Falling of the Leaves "

20. «Miserrimus"

21. "The Priest and the Fairy"

22. "The Fairy Pedant"

23. "She Who Dwelt among the Sycamores"

24. "On Mr. Nettleship's Picture at the Royal Hibernian Academy "

25. "A Legend"

\section{6. "An Old Song Re-sung "}

27. "Street Dancers"

28. "To an Isle in the Water"

29. "Quatrains and Aphorisms "

30. "The Seekers "

« Crossways » (1889), in Poems (1895)

1. "The Song of the Happy Shepherd "

2. "The Sad Shepherd"

3. "The Cloak, the Boat, and the Shoes"

4. "Anashuya and Vijaya"

5. "The Indian Upon God"

6. "The Indian to his Love»

7. "The Falling of the Leaves"

8. "Ephemera»

9. "The Madness of King Goll»

10. "The Stolen Child»

11. "To an Isle in the Water"

12. "Down by the Salley Gardens "

13. "The Meditation of the Old Fisherman "

14. "The Ballad of Father O'Hart "

15. "The Ballad of Moll Magee "

16. "The Ballad of the Foxhunter " 
Pour élaborer le cycle "Crossways ", Yeats a modifié sensiblement la structure du recueil The Wanderings of Oisin and Other Poems. Le regroupement de ses poèmes n'a toutefois rien d'aléatoire. L'artiste « en a soigneusement établi l'ordre. On se doit de les considérer dans les ensembles qu'il a composés. " (Genet 1981, 591) Quel que soit le cycle, tous dialoguent de part et d'autre des trois ‘ poèmes indiens ${ }^{6}$ (ci-dessus en rouge). Dans "Crossways " comme dans "The Wanderings of Oisin ", chaque poème possède à la fois son miroir et son contrechant. Certains ont été repris tels quels, mais déplacés (en bleu), d’autres enfin ont été supprimés. Parmi les seize poèmes qui composent "Crossways », six sont inédits?. "An Old Song Re-Sung ", quant à lui, devient "Down by the Salley Gardens». Il s'agit pourtant du même texte. En remplaçant le titre du poème par l'incipit de la chanson qui l'a inspiré, Yeats souligne la présence d'une émotion qui fut d'abord musicale et suscite - volontairement ou non - le désir de partir à sa recherche. Revenons à elle.

Dans le glossaire du recueil Poems, Yeats donne une précision supplémentaire : « « Down by the Salley Gardens > is an extension of three lines sung to me by an old woman at Ballisodare. „ ${ }^{8}$ Bien que cette note écrite par le poète lui-même soit bien connue, elle est presque toujours amputée de cette précision : "pour moi ». Ainsi une chanson lui a-t-elle été adressée personnellement. Que Yeats ait sollicité le chant ou qu'on le lui ait offert, ni la prestation musicale ni l'écoute ne sont nées du hasard. Cette chanson a été chantée pour lui, afin que, peut-être, il la chantât à son tour. Mais de quelle manière ?

Selon Yeats, le vers possède une musique intrinsèque qu'il faut tirer du silence. "Le pouvoir des mots réside dans leur profération et dans leur audition, qu'elle soit mentale ou réelle. " Des travaux sur la voix de l'acteur à l'Abbey Theatre, l'analysent finement (Longuenesse 2015, 36). Intérieur ou matérialisé, ce ‘ dit ` est déjà un chant, et parfois même une incantation. D'une vieille chanson, Yeats fait un poème dont lui seul connaît la mélodie et, à sa manière, le remet en musique pour qu’à notre tour, nous le disions.

Reprenant symboliquement la lyre des âges anciens, il nous rappelle que la poésie fut d'abord un Chant et le poète un Chanteur. Originellement, la culture irlandaise est une culture de tradition orale, c'est-à-dire une culture sans écriture dont les récits et les mythes ont pu traverser les siècles parce qu'ils ont été chantés, repris et par là même transmis ; mais si Yeats accomplit à son tour le geste du passeur ou du barde, geste commun aux autres pays celtes, le chant de sa lyre reste muet. Le poète chante a cappella pour que se déploie le phrasé musical des mots qu'il a fait sonner - syllabes, consonnes, voyelles, pauses et accents - dans un dialogue subtil avec l'art savant, qui est celui de l'écrit :

Pour Yeats, dès que le discours musical entre en rapport avec le chanté, il sort du rythme linguistique, et prend le rythme musical : il se désaccentue et se réaccentue, [...] C'est par lui-même qu'il doit manifester une musicalité propre, beaucoup plus complexe que la plus subtile des partitions. (Longuenesse 2015, 53) 


\section{"You Rambling Boys of Pleasure "}

Quelle chanson Yeats avait-il entendue à Ballisodare ? Une chanson en gaëlique peut-être ? Lidée était séduisante. Dans une lettre à Katherine Tynan datée de 1888 (Jeffares 1996, 4), Yeats décrit le poème qu'il vient d'écrire comme " an Old Irish verse ». On se prend à rêver aux charmes d'une transmutation opérée par un poète irlandais d'expression anglaise?. Il semble pourtant que cette piste ait dû être abandonnée assez tôt : la chanson d'origine était probablement déjà en langue anglaise lorsque Yeats la découvrit.

Dans les années soixante, le titre d'une ‘ broadside ballad > apparaît dans un article publié par le Trinity College (Shields 1965, 22s) ${ }^{10}$. Il s'agit de "You Rambling Boys of Pleasure ", chanson dont il existe une multitude de variantes. Les avis concordent aujourd'hui pour identifier ce timbre célèbre comme l'élément déclencheur du poème. Que ses paroles soient en anglais n'exclut pas d'emblée qu'il ait pu être un chant transcrit du gaëlique. Or les chansons en gaëlique n'étaient pas imprimées : les musiciens traditionnels ne ressentaient sans doute pas la nécessité de fixer leurs airs sur des ‘ broadsheets `, ces ‘ interface de papier > entre l'Ecrit et l'Oral dont ils devaient se méfier (Atkinson/Steve 2016). Dès lors, comment affirmer avec certitude l'existence d'une source purement irlandaise ? Quoi qu'il en soit, la chanson "You Rambling Boys of Pleasure » a traversé les générations.

Elle fait partie du répertoire enregistré par des groupes folk des années soixante-dix tels que Planxty ou les Chieftains, acteurs du renouveau de la musique populaire irlandaise. S'adressant à son public au début d'un concert, Andy Irvine, qui fut l'un des membres du groupe Plantxy, témoigne d'un ' folk process , plein de fantaisie et avoue, non sans un peu d'humour noir, ne pas avoir eu l'opportunité d'interpréter, du vivant de sa mère, une chanson qu' elle aurait pu lui transmettre, ce qui n'exclut nullement le dialogue post mortem : "I know she is around somewhere, because sometimes I feel her in my brain saying when... I'm a bit nervous, sometimes a bit nervous, and I hear her saying: ^Andrew, don't forget the words s. " ${ }^{11}$

Parmi les innombrables versions qui ont circulé sur les routes du monde anglophone, la version ci-contre figure au catalogue de la Bodleian Library d'Oxford qui met désormais en ligne plusieurs ‘ broadsheets > numérisées. Elle aurait été éditée entre 1780 et 1812 . On observe ici qu'il n'est nullement question d'un jardin.

Dans une version ultérieure citée par Shield, le décor d'un " flowery garden » est posé. Il est le lieu de la rencontre, et bientôt de la perte, comme dans le poème de Yeats :

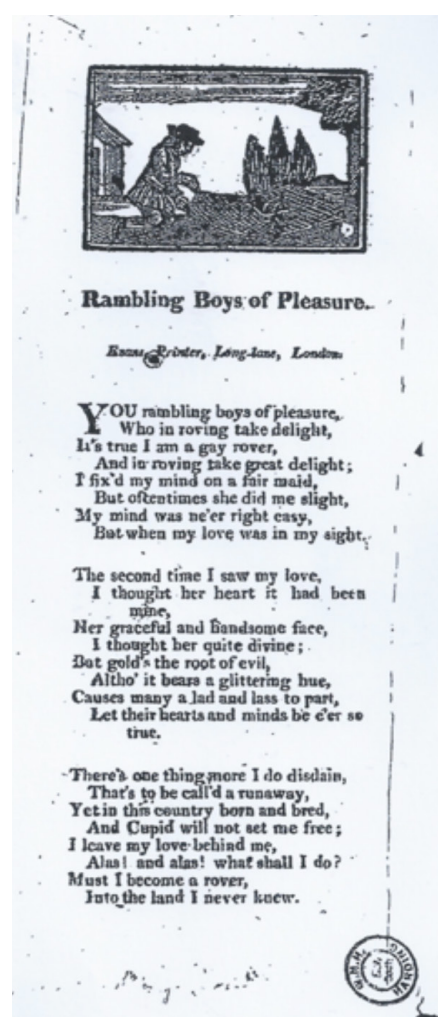

Figure 2. «Rambling Boys of Pleasure ", Broadside Ballads 
Down by yon flowery garden

my love and I we first did meet.

Presque toutes les versions à venir reprendront ces mots-là en ouverture du deuxième couplet. Yeats en fera le vers initial de son poème et prolongera son écho de cloche triste jusqu'au début de la seconde strophe : "In a field by the river, my love and I did stand ".

La version sur laquelle je m’appuie à présent se trouve dans la Frank Kidson Broadside Collection qui en contient plusieurs autres de même facture ${ }^{12}$. Dans ce modèle plus récent (sans doute du milieu du XIX ${ }^{e}$ siècle), le texte s'est allongé jusqu’à former cinq strophes. La deuxième est toujours celle qui est la plus proche du poème de Yeats :

It was down by Sally's garden,

One evening as I chanced to stray,

There I beheld my charmer.

She appeared to me like the Queen of May,

She bid me take love easy,

Just as the leaves fall from the tree;

But I being young and airy,

My love and I could not agree.

En bas, près du jardin, une femme était apparue telle une reine de mai. Dans la chanson populaire, elle se prénommait Sally et ce jardin était le sien. Jusqu'au jour où le poète glissa, par euphonie, du jardin de Sally aux jardins des saules, et occulta son nom. Troublante métamorphose. "The Salley Gardens » de Yeats ne sera plus désormais traversé que par de petits pieds blancs comme neige ( little snow-white feet »). Ses mots se sont gravés dans un temps arrêté. Le < rambling boy > quant à lui, n'est plus qu'un modèle lointain.

\section{Quand la musique reprend ses droits}

À gauche, une version du XIX e siècle diffusée sur une ‘ broadsheet , à droite, l'une de celles que l'on chante aujourd'hui encore. Il s'agit de la fin de "You Rambling Boys of Pleasure ». Les différences entre les deux textes montrent que la chanson a continué d'évoluer en marge de la version imprimée. Le dernier couplet, par exemple, qui est une sorte de retour sur soi un peu mélancolique, est tombé en désuétude. Nul ne le chante plus. Les interprètes d'aujourd'hui terminent tous par ces mots : "Hard fortune would ne'er daunt me / For I am young and the world is wide " qui est désormais la fin > officielle > de la chanson. Ils n'évoquent plus Cupidon, mais en appellent à un avenir radieux dans des lieux évocateurs de l'histoire récente des Irlandais : «Belfast town " (Andy Irvin, Olivia Chaney, Luke McNally), "Americay » ou «America» (Loreena McKenitt, Olliver Mulligan, etc.). 
I wish I was in Dublin,

And my true love along with me,

Money to support us,

And keep us in good company.

Money to support us

And a flowing bowl on every side,

Hard fortune ne'er should daunt us,

For I am young \& the world is wide.
And I wish I was in Belfast town,

And my true love along with me.

With money in my pocket

To keep us in good company.

Liquor to be plenty,

A flowing glass on every side,

Hard fortune would ne'er daunt me,

For I am young \& the world is wide.

And I wish I was in Belfast town,

And my true love along with me.

With money in my pocket

To keep us in good company.

Liquor to be plenty,

A flowing glass on every side,

Hard fortune would ne'er daunt me,

One thing more I do disdain,

That is to be called a run-away.

Now in this country bred $\&$ born

But Cupid did not set me free.

\section{Les mots entrent en résonance}

Aucun prosaïsme dans «Down the Salley Gardens ». Le verbe poétique de Yeats y est volontairement simple, d'une simplicité qu'on pourrait croire empruntée au modèle populaire. La réalité est plus complexe. Ce modèle populaire, nous le connaissons : ballade chantée en bonne compagnie, dans le tintement des verres emplis à ras bord, ballade pour prolonger la route sous les pas du ‘ rover , ballade distendue à loisir, coupée d'intermèdes, et joliment ornée, juste avant le temps.

La simplicité du poème n'est pas du même ordre. Cette simplicité-là est celle d'une épure, le produit d'un art dans lequel la spontanéité est tenue court et le chagrin bridé. L'art de Yeats est soutenu quant à la forme et à la prosodie : deux strophes de quatre vers aux rimes plates que l'on pourrait presque réduire à une sonorité dominante (le [i:] de salley, meet, feet, easy, leaves, tree, meet, agree, field, leaning, weirs, tears, le [æ] plus lourd de stand ou de hand marquant peut-être le poids de la main posée sur l'épaule). Il l'est aussi par la métrique : des vers de 13 pieds, avec une césure $7+6$ qui contraint le poète à ôter la préposition ‘ to ', au phrasal verb < take to > (vers 3 et 6). 
Le poète nous dit peu de choses pour mieux nommer - et par quatre fois - un lieu voué au regret et à la mélancolie. Ce lieu résonne, impossible à oublier : des jardins où poussaient des saules. Dans l'imaginaire anglais, le saule (et en particulier le saule pleureur, ‘ the weeping willow >), est l'un des symboles des amours malheureuses et du deuil. Pourtant, le mot ( saule > se dit ( willow > en anglais, et non ( salley >. C'est le rapprochement du mot ‘ salley ) avec le gaëlique « saileán ` qui le fait comprendre comme l'équivalent du mot « saule $>^{13}$.

Sur le plan purement formel, « Down by the Salley Gardens » est en effet volontairement laconique. Des répétitions de mots et d'idées concourent à une économie de moyens que l'on peut aussi visualiser :

Down by the Salley Gardens, my love and I did meet.

She passed the Salley Gardens, with little snow-white feet.

She bid me take love easy, as the leaves grow on the tree;

But I was young and foolish, with her did not agree

In a field by the river, my love and I did stand,

And on my leaning shoulder, she laid her snow-white hand.

She bid me take life easy, as the grass grows on the weirs;

But I was young and foolish, and now I am full of tears.

Les images sont rares et choisies, toujours évocatrices et placées en regard (ou, si l'on veut, en miroir) : « little swow-white feet/little swow-white hand "; " the leaves grow on the tree " (" les feuilles poussent sur l'arbre", qui est au singulier) ; " the grass grows on the weirs " (" l'herbe pousse sur les retenues d'eau "). Car de même que les nombres s'équilibrent et que les éléments se répondent : le bois et l'eau, le figé et le fluide, le lent et le vif, une langue savante - l'anglais - trace son chemin dans le pays perdu de l'oralité.

On le voit, la construction de «Down by the Salley Gardens » est si serrée que le message traverse les deux strophes, enjambant les répétitions comme on franchit la rivière sur un pont de bois jeté en travers, ce qui donne à la compréhension rapide : «My love bid me to take life easy, but I was young and foolish, with her did not agree, and now I am full of tears. " (" Mon amour m’a offert de prendre la vie simplement, mais j’étais jeune et insensé, avec elle je n'étais pas d'accord, et maintenant je suis plein de larmes. ") S'ensuit un déroulement des causes et des conséquences parfaitement lisibles, une efficacité qui sied à la langue orale et plus encore à la langue chantée : quelque chose d'aussi frais et d'aussi simple, en apparence, que la mélodie populaire dont le poème est issu. 


\section{"The Maids of Mourne Shore » ou le rapt d'une mélodie}

" Down by the Salley Gardens » aurait pu demeurer cette " Old Song Re-sung », cette vieille chanson réinventée, une chanson avec des paroles autres, certes, mais figées désormais dans un recueil, autrement dit des paroles avec une musique fantôme, puisque la mélodie était enclose dans la tête du poète, et de lui seul. L'histoire de "Sally Gardens ", on le sait, ne s'arrête pas à la ‘ recréation > de Yeats : après avoir été inspiré par une chanson, son texte est devenu, quelques années plus tard, un poème mis en musique, mais sur un air différent du modèle présumé, un air qui ‘ colle > toutefois parfaitement au poème. C'est ainsi que le grand public le connaît aujourd'hui. Récapitulons.

"Down by the Salley Gardens " fut tout d'abord un poème inspiré par une chanson, puis une chanson " ni tout à fait la même ni tout à fait une autre $»^{14}$ dont les paroles renouvelées ont à leur tour fait naitre un désir de musique irrépressible. À ce moment du récit, vont se rencontrer deux réalités qui auraient pu se neutraliser l'une l'autre : un compositeur, auquel est venue l'idée de plaquer une mélodie sur les paroles de Yeats - en l'occurrence, Herbert Hughes en 1909 - et une mélodie qui n'est plus celle de "You Rambling Boys of Pleasure " (chanson de référence pour Yeats) mais celle d'une autre chanson traditionnelle : "The Maids of Mourne Shore », dont Hughes s'est emparé.

Des paroles de William Butler Yeats sur un air irlandais que le compositeur Herbert Hughes a adapté sur elles, est-ce réellement cela, "Salley Gardens » ? Oui, en un sens, puisque cette version-là - paroles de Yeats et mélodie populaire quasi indissociable de ces paroles s'est universellement imposée, y compris au compositeur Benjamin Britten qui en fait une version pour voix et piano sans modifier la mélodie choisie par Hughes.

On peut maintenant lire sur certaines éditions modernes : " ‘ Sally Gardens , lyrics by W.B. Yeats, an Irish air arranged by Herbert Hughes ». Ce mariage heureux a eu quelques conséquences : les paroles de "The Maids of Mourne Shore " ne sont presque plus jamais chantées sur leur mélodie d'origine, alors qu'elles ne manquent pas de charme ${ }^{15}$. On entend aussi souvent l'air de "The Maids of Mourne Shore " sans aucune parole, de sorte que ce sont celles de "Salley Gardens » qui viennent le plus spontanément à l'esprit. "The Maids of Mourne Shore » a donc en quelque sorte donné sa mélodie à « Salley Gardens ».

La mélodie de Hughes s'est même si fortement arrimée au poème de Yeats que les autres mélodies reprenant les paroles de "Sally Gardens " sonnent presque comme des bizarreries. C'est le cas des très jolies versions antérieures de John Ireland (1879-1962) et surtout de la femme compositeur Rebecca Clarke (1886-1979). Force est de constater que c'est « Down by the Salley Gardens » dans la version Yeats-Hughes qui a séduit le plus grand nombre d'interprètes et d'arrangeurs dans tous les styles. Cette extraordinaire bonne fortune est la preuve d'une adéquation parfaite entre la mélodie choisie par Hughes et la prosodie du texte que nous avons schématisée ainsi : 


\section{Mélodie 1}

Down I by the salley I gardens, my I love and I did I meet. (3 temps tenus)

She I passed the Salley I Gardens, with I little snow-white I feet. (idem)

\section{Mélodie 2}

She I bid me take life I easy, as the I leaves grow on the I tree; (id.)

-

Mélodie 1 (reprise)

But I I was young and I foolish, with I her did not a- I gree. (id.)

○

○

Figure 3. Eléments de prosodie mis en valeur par la mélodie de Herbert Hughes

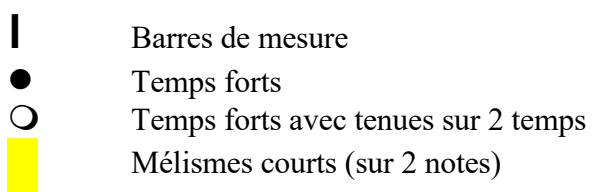

\section{En bleu}

Départ sur une levée (anachrouse)

En rouge

Figuralisme : mélisme resserré sur " grow on the »

Soulignement

Amplification mélodique (sur « She bid me take life easy ») 


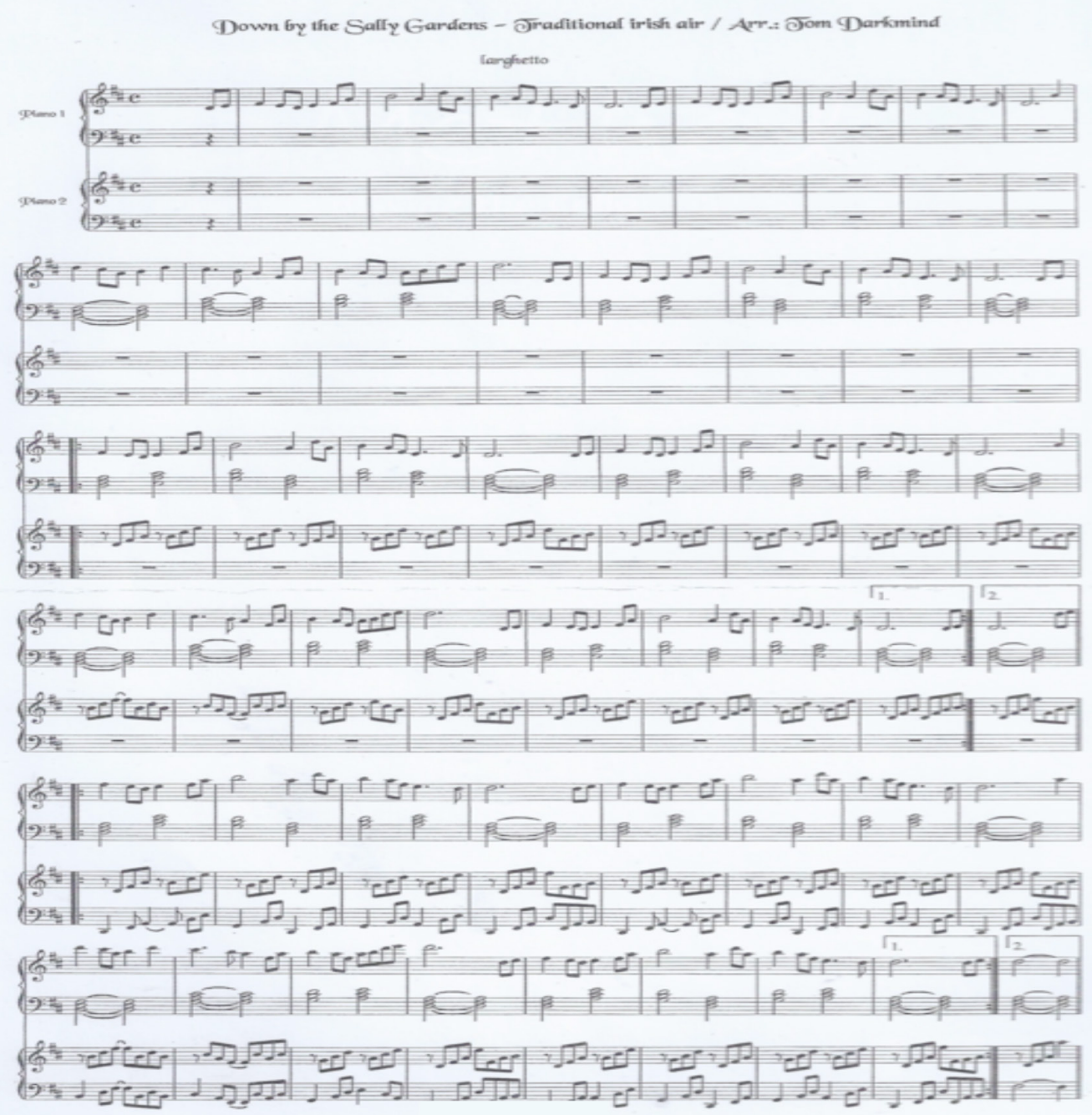

Figure 4. «Down by the Sally Gardens », Arrangement Tom Darkmind, http://fr.scorer.com/I/Partitions/300120626.html

Dans une lettre à Paul Demeny datée du 15 mai 1871, dite "Lettre du Voyant ", Arthur Rimbaud s'en prend aux Romantiques " qui prouvent si bien que la chanson est si peu souvent l'œuvre, c'est-à-dire la pensée chantée et comprise du chanteur ». Ainsi, l'œuvre est-elle à la fois une pensée chantée et une pensée comprise. Mais comprise par qui ? En faisant intervenir une autre dimension, celle de la perception sensorielle, c'est-à-dire en l'occurrence, la dimension de l'écoute, tout devient plus simple. Rimbaud place quelqu'un d'autre en face du chanteur. Cet autre, ce pourrait être l'auditeur, ce lecteur qui lit en écoutant. Que l'on déroule complètement le raisonnement rimbaldien, et l'on obtient la relation suivante : la 
chanson est très exactement l'œuvre chantée et comprise $d u$ chanteur lorsqu'elle est chantée pour un tiers, qui la reçoit.

Par ailleurs, si l'on veut bien se souvenir que le poème, dans l'histoire, a d'abord été chanson, le mot ، chanson > peut aisément être remplacé par le mot ‘ poème `. C'est pourquoi je ne crois pas trop m'avancer en disant que la chanson, qui est «l'œuvre pensée et comprise du chanteur " peut parfois se confondre avec le poème lorsqu'il est l'œuvre pensée et comprise du Poète, au temps où il chantait encore. N'est-ce pas la nostalgie de cet état ancien, la nostalgie de ce « Perdu » dont parle Pascal Quignard (Les Larmes, 2016), qui conduisit la main de Yeats dans sa hâte à jeter sur le papier « le vierge, le vivace et le bel aujourd'hui ${ }^{16}$ que le poète moderne, devenu scribe, ne chante plus ?

\section{Notes}

1 Anne-Marie Gouiffès est professeur agrégé d'éducation musicale et de chant choral au collège Stanislas (Paris). Docteur ès musicologie, elle a été membre de l'Observatoire musical français jusqu'à l'intégration de ce laboratoire au sein de l'IREMUS (2014). Ses travaux portent sur l'interprétation, les techniques vocales et la prosodie d'une part, sur les politiques culturelles - en particulier celles des théâtres lyriques - d'autre part (cf. PhD 2000).

2 Ballisodare est le nom local de Ballysadare, village situé à environ sept kilomètres de Sligo, dans le Nord-Ouest de l'Irlande.

3 C'est nous qui soulignons.

4 Dans Poems, «The Wanderings of Oisin » est en effet devenu « The Wanderings of Ushee ».

5 Les deux premiers textes sont des pièces de théâtre.

6 "Anashuya and Vijaya ", qui apparaitt pour la première fois dans "Crossways » a la forme d'un duo destiné à être chanté pour moitié. Anashuya et Vijaya forment un couple. La femme (Anashuya) s'exprime en parlant, l'homme en chantant (cf. l'indication de jeu : "VIJAYA sings»).

7 "The Song of the Happy Shepperd ", «The Sad Shepperd " « The Cloak, the Boat, and the Shoes ", "The Madness of King Goll », "The Ballad of Father O'Hart » et "The Ballad of the Foxhunter ».

8 Cf. l'édition originale de 1895, 283 : « une extension de trois lignes chantées pour moi par une vieille femme à Ballisodare ». C'est nous qui soulignons.

9 Un parallèle pourrait être fait avec ces écrivains bretons d'expression française, à l'image du poète Guillevic, écrivains pour lesquels « la perte de la langue [originelle] est vécue comme un manque qui n'est pas dit nécessairement, mais qui active, sur le mode de la tension, leur pratique du français comme langue d'écriture " (cf. Gontard 2002).

10 Selon Shields 1965, le texte des ، broadside ballads , dont les airs étaient connus de tous, était imprimé sur le recto d'une feuille appelée ` broadsheet qui était tirée à plusieurs milliers d'exemplaires et vendue dans la rue.

11 Dans cette vidéo de 2015, Irvine dit encore : «I've always had the desire to tell audiences how I learned these Antrim songs at my mother's knee, but unfortunately that did not occur because my mother was a musical comedy actress. " ("J'ai toujours eu envie de raconter à mon public 
comment j'avais appris cette chanson sur les genoux de ma mère, mais malheureusement, ça ne s'est pas passé ainsi parce que ma mère était une actrice de comédie musicale. ") https://www. youtube.com/watch?v=plXL0aTh9Hg (consultation 02.2019).

12 Cf. Frank Kidson Broadside Collection, vol. 10, 252. Frank Kidson (1855-1926) était un collectionneur érudit, passionné de musique populaire. Il entreprit de rassembler les premiers « broadsheets ', dans les années 1880. On peut également avoir accès à la version numérisée de "You Rambling Boys of Pleasure » en se rendant sur le site de la Vaughan Williams Memorial Library : http://www.vwml.org.uk/ (consultation 02.2019).

13 Le mot ` saule > se dit ` saileán > en gaëlique.

14 Paul Verlaine, "Mon rêve familier ".

15 Voici le premier couplet de "The Maids of Mourne Shore » : "Ye hills and dales and flowery vales that lie around Mourne shore / Ye winds that blow over Martin's hills will I never hear you more / Where the primrose grows and the violet blows and the sporting trout there plays / With line and hook, delight I took to spend my youthful days. "

16 Stéphane Mallarmé, « Le vierge, le vivace et le bel aujourd’hui » (sonnet dit ‘ du cygne `).

\section{Bibliographie}

Atkinson, David / Roud, Steve : Street Ballads in Nineteenth-Century Britain, Ireland and North America. The Interface between Print and Oral Traditions. Londres/New York : Routledge, 2016.

Genet, Jacqueline : Les fondements et l'évolution de la création poétique. Lille : Presses Universitaires du Septentrion, 1981.

Gontard, Marc : "Le deuil de la langue. Littérature bretonne de langue française ». In : Cabiers de sociolinguistique 7,1 (2002), 179-193.

Jeffares, A. Norman : A Commentary on the Collected Poems of W.B. Yeats. London : Macmillan \& Co, 1968.

Jeffares, A. Norman : Yeats's Poems. UK : Macmillan Press, 1996.

Longuenesse, Pierre : Yeats et la scène. L'acteur et sa voix à l'Abbey Theatre de Dublin. Lille : Presses Universitaires du Septentrion, 2015.

Mallarmé, Stéphane : Poésies. Bruxelles : Edmond Deman, 1899.

Quignard, Pascal : Les Larmes. Paris : Gallimard, 2016.

Raine, Kathleen : Yeats the Initiate. Essays on Certain Themes in the Work of W. B. Yeats. Mountrath/ London : George Allen \& Unwin Limited, 1986.

Rimbaud, Arthur : Une saison en enfer. Bruxelles : Alliance typographique (M.-J. Poot et Compagnie), 1873.

Rimbaud, Arthur : Poésies complètes. Paris : Léon Vanier Libraire-Editeur, 1895.

Rimbaud, Arthur : "Lettre à Paul Demeny du 15 mai 1871 ». In : La Nouvelle Revue Française 8 (1912) : Trois lettres inédites d'Arthur Rimbaud, 568-580.

Shields, Hugh E. : "Yeats and the ‘ Salley Gardens > ". In : Hermathena 101 (autumn 1965) : Yeats Number, 22-26. 
Verlaine, Paul : Poèmes saturniens. Paris : Alphonse Lemerre, 1866.

Yeats, William Butler : The Wanderings of Oisin and Other Poems. London : Kegan Paul \& Company, 1889.

Yeats, William Butler : Poems. London \& Boston : T. Fisher Unwin/Copeland and Day, 1895.

Yeats, William Butler : Choix de poèmes. Paris : Aubier, ${ }^{3} 1990$.

\section{Sitographie}

- Archives

Broadside Ballads Online, http://ballads.bodleian.ox.ac.uk/ (consultation 02.2019).

Frank Kidson Broadside Collection, https://www.vwml.org/archives-catalogue/FK (consultation 01.2019).

The Vaughan Williams Memorial Library, http://www.vwml.org.uk/ (consultation 02.2019).

- Partition

"Down by the Sally Gardens » (arrangement Tom Darkmind), http://fr.scorer.com/I/Partitions/ 300120626.html (consultation 02.2019).

- Sources audiovisuelles

Irvine, Andy : "You Rambling Boys of Pleasure ", https://www.youtube.com/watch?v=plXL0aTh9Hg (consultation 07.2018).

McKenitt, Loreena : " Down by the Salley Gardens », https:/www.youtube.com/watch?v=9SAH79tM-lc (consultation 05.2018).

Scholl, Andreas : " Down by the Salley Gardens », https://www.youtube.com/watch?v=QBWhhsnpRAc (consultation 04.2017).

The North Texas Gaelic Young Chorus : «Gort na Saileán », https:/www.youtube.com/ watch?v=1qCMShuGFmM (consultation 09.2018).

\section{Discographie sélective}

Auger, Arleen / Baldwin, Dalton : Love Songs. Delos DE 3029, 1992 (CD).

Deller, Alfred / Deller, Mark / Dupré, Desmond : Folksongs. Harmonia Mundi HMD 226, 1972 (33 tours).

Kronos Quartet : Folk Songs. Nonesuch 559151-2, 2017 (CD).

McKenitt, Loreena : "Ye Rambling Boys of Pleasure ». In : The Chieftains : Tears of Stone. RCA Victor/BMG Classics - 0902668968 2, 1999 (CD).

Planxty : After the Break. Tara 3001, 1979 (33 tours).

Terfel, Bryn / Martineau, Malcolm : Silent Noon. Deutsche Grammophon 00289474 2192, 2004 (CD). 